\title{
Normal State of Highly Polarized Fermi Gases: Simple Many-Body Approaches
}

\author{
R. Combescot \\ Laboratoire de Physique Statistique, Ecole Normale Supérieure, 24 rue Lhomond, 75231 Paris Cedex 05, France
}

A. Recati and C. Lobo

Dipartimento di Fisica, Università di Trento and CNR-INFM BEC Center, I-38050 Povo, Trento, Italy

F. Chevy

Laboratoire Kastler Brossel, Ecole Normale Supérieure, 24 rue Lhomond, 75231 Paris Cedex 05, France

(Received 16 February 2007; published 1 May 2007)

\begin{abstract}
We consider the problem of a single $\downarrow$ atom in the presence of a Fermi sea of $\uparrow$ atoms, in the vicinity of a Feshbach resonance. We calculate the chemical potential and the effective mass of the $\downarrow$ atom using two simple approaches: a many-body variational wave function and a $T$-matrix approximation. These two methods lead to the same results and are in good agreement with existing quantum Monte Carlo calculations performed at unitarity and, in one dimension, with the known exact solution. Surprisingly, our results suggest that, even at unitarity, the effect of interactions is fairly weak and can be accurately described using single particle-hole excitations. We also consider the case of unequal masses.
\end{abstract}

DOI: 10.1103/PhysRevLett.98.180402

The investigation of ultracold Fermi gases with two unbalanced hyperfine states (which we shall denote as $\uparrow$ and $\downarrow$ ) has been through an impressive expansion last year. This subfield is of great interest both on practical and on theoretical grounds. Indeed, on one hand, it is related to other fields of physics, namely, superconductivity, astrophysics, and high-energy physics, where similar situations arise [1]. On the other hand, the additional parameter provided by the population imbalance should provide a tool to deepen our understanding of the Bose-Einstein condensation (BEC)-BCS crossover in these systems and contribute to improving our control of many-body theory. This recent activity has been started by striking experimental results [2] which have given rise to a considerable number of theoretical works [3]. Experiments performed on trapped systems have observed equal density superfluid states as well as partially and fully polarized regions.

The analysis of the $T=0$ phase separation requires the knowledge of the properties of both the superfluid and the partially polarized normal phase [4-6]. This has been developed in the recent work of Lobo et al. [6], where, at unitarity, the properties of the partially polarized phase have been obtained by calculating through a quantum Monte Carlo (QMC) approach the parameters which characterize a single $\downarrow$ atom immersed in a Fermi sea of $\uparrow$ atoms, with density $n_{\uparrow}=k_{F}^{3} /\left(6 \pi^{2}\right)$. In this way, they were able to obtain very good agreement with experimental results.

Here we consider the general problem of a single $\downarrow$ atom in a completely polarized $\uparrow$ atom Fermi sea. The $\uparrow-\downarrow$ interaction is characterized by an $s$-wave scattering length $a$, whose value can be tuned via a Feshbach resonance from the BEC $\left(1 / k_{F} a \gg 1\right)$ to the BCS $\left(1 / k_{F} a \ll-1\right)$ limits. The Fermi gas is ideal due to the suppression of higher angular momentum scattering at low temperatures. This problem is a much simpler one than the case of two equal
PACS numbers: 05.30.Fk, 03.75.Ss, 71.10.Ca, 74.72.-h

spin populations in the BEC-BCS crossover, although still quite nontrivial due to the absence of a small parameter in the strongly interacting regime. It is the simplest realization of the moving impurity problem, and it bears a strong similarity with other old, famous, and notoriously difficult condensed matter problems, such as the Kondo problem, the x-ray singularity in metals [7], and the mobility of ions [8] and ${ }^{4} \mathrm{He}$ atoms [9] in ${ }^{3} \mathrm{He}$. This gives to the present atomic system a much wider significance beyond the context of polarized Fermi gases. We may hope to have a full control and understanding of this system and to obtain further physical insight in these kinds of problems.

In this Letter, in addition to considering a general scattering length $a$, we will extend the parameter range by treating the general case where the masses $m_{\uparrow}$ and $m_{\downarrow}$ are different, although they have been the same in experiments up to now. This can be accomplished by using atoms belonging to different elements. The limit $m_{\downarrow} / m_{\uparrow} \rightarrow \infty$ is then compared to the fully solvable problem of a fixed impurity in a Fermi sea.

Here we are interested in the two physical quantities which have been calculated by Lobo et al. [6], namely, the chemical potential $\mu_{\downarrow}$ of the $\downarrow$ atom and its effective mass $m^{*}$. We have addressed this problem by two different and complementary many-body methods. The first one is a natural extension of the many-body trial wave function used by Chevy [5] to obtain the effective mass. The second one is a $T$-matrix approximation, whose basic ingredient, ladder diagrams, appears in more elaborate schemes. This approximation has already been used in a wide range of physical systems, including high $T_{c}$ superconductors [10] and the BEC-BCS crossover [11,12], and it is known to give reasonable results. The variational method has the advantage of providing a rigorous upper bound to the energy, while the $T$ matrix can be easily extended to 
include more sophisticated approximations. As we will see, these two methods lead exactly to the same results and, moreover, are in surprisingly good agreement with QMC calculations [6,13].

The trial wave function $|\psi\rangle$ we consider, for a system of total momentum $\mathbf{p}$, is the following momentum eigenstate (we set $\hbar=1$ throughout the Letter):

$$
|\psi\rangle=\phi_{0}|\mathbf{p}\rangle_{\downarrow}|0\rangle_{\uparrow}+\sum_{q<k_{F}}^{k>k_{F}} \phi_{\mathbf{q k}}|\mathbf{p}+\mathbf{q}-\mathbf{k}\rangle_{\downarrow} c_{\mathbf{k}_{\uparrow}}^{\dagger} c_{\mathbf{q}_{\uparrow}}|0\rangle_{\uparrow},
$$

where $c_{\mathbf{k}_{\uparrow}}$ and $c_{\mathbf{k}_{\uparrow}}^{\dagger}$ are annihilation and creation operators, respectively. In the first term, the $\uparrow$-spin free Fermi sea is in its ground state $|0\rangle_{\uparrow}=\prod_{k<k_{F}} c_{\mathbf{k}_{\uparrow}}^{\dagger}|\mathrm{vac}\rangle$, and the $\downarrow$-spin atom is in the plane-wave state $|\mathbf{p}\rangle_{\downarrow}$, while in the second term it is in excited states corresponding to the creation of a particlehole pair in the Fermi sea with momentum $\mathbf{k}$ and $\mathbf{q}$, respectively, the $\downarrow$-spin atom carrying the rest of the momentum. The coefficients $\phi_{0}$ and $\phi_{\mathbf{q k}}$ are found by minimizing the total energy. This wave function is by construction suitable to reproduce the molecule in the BEC limit as well as the perturbative mean-field limit. We follow the procedure of Ref. [5], in particular, with respect to the zero range interaction and the regularization in terms of the scattering length. We obtain for the change in energy $E$ due to the addition of the $\downarrow$-spin atom:

$$
\begin{aligned}
E= & \epsilon_{\downarrow p}+\sum_{q<k_{F}}\left[\frac{m_{r}}{2 \pi a}-\sum_{k} \frac{2 m_{r}}{k^{2}}\right. \\
& \left.+\sum_{k>k_{F}} \frac{1}{\epsilon_{\uparrow k}+\epsilon_{\downarrow \mathbf{p}+\mathbf{q}-\mathbf{k}}-\boldsymbol{\epsilon}_{\uparrow q}-E}\right]^{-1},
\end{aligned}
$$

where $\epsilon_{\uparrow, l k}=k^{2} / 2 m_{\uparrow, \downarrow}$ is the kinetic energy of the $\uparrow$ and $\downarrow$ atoms, and $m_{r}=m_{\uparrow} m_{\downarrow} /\left(m_{\uparrow}+m_{\downarrow}\right)$ is the reduced mass. For $\mathbf{p}=\mathbf{0}$, we have $E=\mu_{\downarrow}$, while the variation of $E$ for small p gives the effective mass.

These results can be obtained exactly from the knowledge of the self-energy $\Sigma(p, \omega)$ of the $\downarrow$ atom. Indeed, the pole of the $\downarrow$ Green's function $G_{\downarrow}(p, \omega)=\left[\omega-\epsilon_{\downarrow p}+\right.$ $\left.\mu_{\downarrow}-\Sigma(p, \omega)\right]^{-1}$, giving the dispersion relation of the $\downarrow$ quasiparticle, satisfies

$$
\omega-\epsilon_{\downarrow p}+\mu_{\downarrow}-\Sigma(p, \omega)=0 .
$$

Since $\omega$ is the energy measured from the chemical potential, and physically the chemical potential corresponds to the addition of a particle with zero momentum $p=0$, the chemical potential is given by

$$
\mu_{\downarrow}=\Sigma(0,0) .
$$

Moreover, the effective mass, giving the dispersion relation of the $\downarrow$ quasiparticle, is obtained from the small $p$ behavior of the dispersion relation and is given by

$$
\frac{m^{*}}{m_{\downarrow}}=\frac{1-\frac{\partial \Sigma}{\partial \omega}}{1+\frac{\partial \Sigma}{\partial\left(p^{2} / 2 m_{\downarrow}\right)}},
$$

where the derivatives of the self-energy are taken for $p=$ $\omega=0$ ( $\Sigma$ is real in the situations we deal with below).

The self-energy itself is obtained [14] from the unknown two-particle vertex $\Gamma$, the only very important simplifying feature of our problem being that the $\uparrow$-atom Green's function is exactly the bare one, namely, $G_{0 \dagger}(k, \omega)=[\omega-$ $\left.\epsilon_{\uparrow k}+\mu_{\uparrow}\right]^{-1}$, where $\mu_{\uparrow}=k_{F}^{2} / 2 m_{\uparrow}$, since the single $\downarrow$ atom does not perturb in the thermodynamic limit the free Fermi sea of $\uparrow$ atoms. To obtain actual answers, we proceed to take the simplest approximation for this vertex, namely, the $T$-matrix approximation, where the $\downarrow$ atom interacts only with a single $\uparrow$ atom. This implies, in particular, that the only excited states of the $\uparrow$ Fermi sea coming in this problem are the single particle-hole excitations, just as in Eq. (1). In this approximation, the $\downarrow$ and the $\uparrow$ atoms scatter any number of times through the bare potential, just as in any two-body problem solved exactly by perturbation theory, which leads to the well-known series of "ladder" diagrams. When there is a single $\uparrow$ atom, the problem reduces to the full scattering of the $\downarrow$ and $\uparrow$ atoms, and the solution can be expressed in terms of the scattering amplitude. When this problem is compared to the one in the presence of the Fermi sea of $\uparrow$ atoms, the bare interaction $V$ can be eliminated in favor of the scattering properties. In our approximation, the vertex $\Gamma$ depends only on the total momentum $\mathbf{K}$ and the total energy $\Omega$ of incoming particles. After performing the above steps, we find $[12,14]$

$$
\begin{aligned}
\Gamma^{-1}(K, \Omega)= & \frac{m_{r}}{2 \pi a}-\int \frac{d \mathbf{k}}{(2 \pi)^{3}}\left[\frac{2 m_{r}}{k^{2}}\right. \\
& \left.+G_{\downarrow}\left(k, \Omega+\mu_{\uparrow}-\epsilon_{\uparrow \mathbf{K}-\mathbf{k}}\right) \theta\left(\epsilon_{\uparrow \mathbf{K}-\mathbf{k}}-\mu_{\uparrow}\right)\right],
\end{aligned}
$$

where $\theta(x)$ is the Heaviside function. In obtaining this result, we have made explicit use of the fact that we will find $\mu_{\downarrow}<0$ as it is obvious physically.

In our approximation, the self-energy is given by

$$
\Sigma(p, \omega)=\frac{1}{2 i \pi} \int \frac{d \mathbf{K}}{(2 \pi)^{3}} \int_{C} d \Omega \Gamma(K, \Omega) G_{0 \dagger}(\mathbf{K}-\mathbf{p}, \Omega-\omega),
$$

where, as mentioned above, using $G_{0 \uparrow}$ as the $\uparrow$-atom Green's function does not imply any approximation. Contour $C$ goes anticlockwise around the left-hand side part $\operatorname{Re} \omega<0$ of the $\omega$ complex plane. We deform it to enclose only the singularities of the integrand. Physically, the singularities of $\Gamma(K, \Omega)$ correspond to the continuous spectrum of the scattering states of the $\uparrow$ and $\downarrow$ atom and possibly to a bound state of these atoms. On the other hand, $G_{0 \uparrow}(k, \omega)$ has just, for fixed $k$, a pole at $\omega=\epsilon_{\uparrow k}-\mu_{\uparrow}$. Since we work at $T=0$, the particle-particle continuous spectrum does not contribute as it is physically obvious. Indeed, from Eq. (6), the corresponding singularities are at $\Omega>0$ and are outside contour $C$. On the other hand, we will restrict ourselves mainly to the case where there are no bound states between the $\uparrow$ and the $\downarrow$ atoms. Such a bound 
state exists clearly in the BEC limit $1 / k_{F} a \rightarrow+\infty$, where molecules will be present. While, as we shall shortly show, our simple approach recovers also this limiting behavior, the effect of a bound state in the intermediate regime will be investigated in further work. This leaves us only with the contribution of the pole of $G_{0 \uparrow}$ :

$$
\begin{aligned}
\Sigma(p, \omega) & =\int \frac{d \mathbf{K}}{(2 \pi)^{3}} \theta\left(\mu_{\uparrow}-\epsilon_{\uparrow \mathbf{K}-\mathbf{p}}\right) \Gamma\left(K, \omega+\epsilon_{\uparrow \mathbf{K}-\mathbf{p}}-\mu_{\uparrow}\right) \\
& =\frac{1}{2 \pi^{2}} \int_{0}^{k_{F}} d K K^{2}\left\langle\Gamma\left(\mathbf{K}+\mathbf{p}, \omega+\epsilon_{\uparrow K}-\mu_{\uparrow}\right)\right\rangle,
\end{aligned}
$$

where the bracket is for the angular average over the direction of $\mathbf{K}$.

At this stage, it is interesting to consider the weak coupling limit $a \rightarrow 0_{-}$, in which case the first term dominates in the right-hand side of Eq. (6). This gives $\Gamma(K, \Omega)=2 \pi a / m_{r} \quad$ and $\quad \Sigma(p, \omega)=\Sigma(0,0)=\mu_{\downarrow}=$ $k_{F}^{3} a /\left(3 \pi m_{r}\right)=2 \pi n_{\uparrow} a / m_{r}$. Hence, we recover the expected result for the mean-field interaction energy with a short-range interaction $V(\mathbf{r})=\left(2 \pi a / m_{r}\right) \delta(\mathbf{r})$. We can view the more general result Eq. (8) as having a similar physical interpretation but with the effective interaction having now a wave vector and energy dependence.

In the general case, Eq. (8) together with Eq. (6) provide an integral equation for $\Sigma(p, \omega)$. We leave the exact solution of this equation for further work, whereas in the present Letter we will proceed to a further approximation. We will just stop at the first step of an iteration loop which would provide the full answer; that is, we will set $\Sigma(p, \omega)=0$ in the expression of $G_{\downarrow}(p, \omega)$. In this case, we can check that Eqs. (2) and (3) are identical, provided $E$ is identified with $\omega+\mu_{\downarrow}$.

For the case of the $\downarrow$ atom chemical potential, we are just left with solving numerically an equation for $\mu_{\downarrow}$. At unitarity, $1 / k_{F} a=0$, and for $m_{\downarrow}=m_{\uparrow}$ this gives $\mu_{\downarrow}=$ $-0.6066 \mu_{\uparrow}$, in remarkable agreement with the QMC [5,6] result $\quad \mu_{\downarrow}=-(3 / 5)(0.97 \pm 0.02) \mu_{\uparrow}=-0.58 \pm$ $0.01 \mu_{\uparrow}$. This surprising agreement suggests that the effect of interactions is weak even at unitarity.

It is interesting to investigate the regime where the ratio $\rho=\left|\mu_{\downarrow}\right| / \mu_{\uparrow}$ becomes large. In this case, the expression of $\Gamma(K, \Omega)$ becomes quite simple. We find to dominant order $\Gamma(K, \Omega)=\left[2 \pi /\left(m_{r} k_{F}\right)\right]\left\{1 / k_{F} a-[\rho r /(1+r)]^{1 / 2}\right\}^{-1}$, with $r=m_{\downarrow} / m_{\uparrow}$. Since $\Gamma$ does not depend on $K$ and $\Omega$, the situation is completely analogous to the one found above in the weak coupling limit: $\mu_{\downarrow}=\Sigma(0,0)$ is easily calculated, and the resulting relation between $\rho$ and $1 / k_{F} a$ can be written as

$$
\frac{1}{k_{F} a}=\sqrt{\frac{\rho r}{1+r}}-\frac{2}{3 \pi} \frac{1+r}{\rho r} .
$$

In the case of equal masses $m_{\uparrow}=m_{\downarrow}$, this is plotted for comparison in Fig. 1 and is seen to give a quite good agreement with the numerical value [15]. In the weak coupling regime $a \rightarrow 0_{-}$, we recover the mean-field result given above, while the asymptotic behavior for large $\rho$ is $\rho=(1+1 / r)\left(1 / k_{F} a\right)^{2}$, which is the two-body bound state energy. This formula can be seen as an interpolation between these two extremes. At unitarity, it gives $\rho=$ $(1+1 / r)(2 / 3 \pi)^{2 / 3}$. For equal masses, we get $\rho \approx 0.71$, which is fairly near the numerical result.

In the inset in Fig. 1, we present the results of our model at unitarity as a function of the mass ratio $r=m_{\downarrow} / m_{\uparrow}$. Again the interpolation equation (9), i.e., $\rho=(2 / 3 \pi)^{2 / 3} \times$ $(1+1 / r)$, is in quite reasonable agreement with numerical results. For small $r$, the chemical potential $\mu_{\downarrow}$ goes to $-\infty$, as can be seen easily from Eq. (8). In the other limit $m_{\downarrow} \rightarrow \infty$, this ratio is seen to saturate.

Assuming that the thermodynamic and infinite mass limits commute, the problem reduces to that of an impurity interacting with a free Fermi sea, which is well known in solid state physics [16]. It can be solved exactly in the following way. The Fermi sea is enclosed in a large sphere of radius $R$, with $R \rightarrow \infty$ in the thermodynamic limit and the impurity at the center. Since the $s$-wave functions have to be zero at the sphere, the allowed wave vectors $k_{p}$ are given by $k_{p} R+\delta_{0}\left(k_{p}\right)=p \pi$, with integer $p \leq n$ and $k_{F} R=n \pi$. For low energy atoms, the phase shift is given by $\tan \delta_{0}(k)=-k a$. The energy of all of the atoms of the Fermi sea is $E=\sum_{p} k_{p}^{2} / 2 m_{\uparrow}$. The calculation is conveniently performed by finding the change in energy due to a change in the scattering length. In this limiting case, the change in total energy of the Fermi sea is identified with the $\downarrow$ atom chemical potential. We find in this way

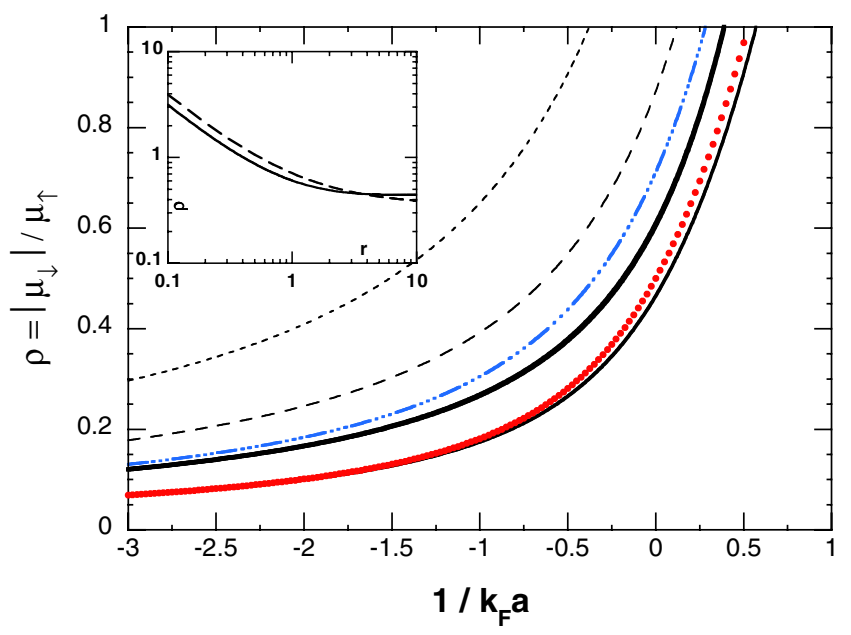

FIG. 1 (color online). Reduced $\downarrow$ atom chemical potential $\left|\mu_{\downarrow}\right|$ as a function of $1 / k_{F} a$ for various mass ratios $r=m_{\downarrow} / m_{\uparrow}$. Black curves from top to bottom: Numerical results for $r=0.25,0.5,1$ (solid thick line), and $\infty$ (lower solid line). The dashed-tripledotted blue line above the $r=1$ line is the corresponding interpolating approximation Eq. (9). The dotted red line just above the $r=\infty$ line is the exact result Eq. (10). The inset compares, at unitarity, the approximation equation (9) (dashed line) with the numerical results (solid line) as a function of the mass ratio $r$. 


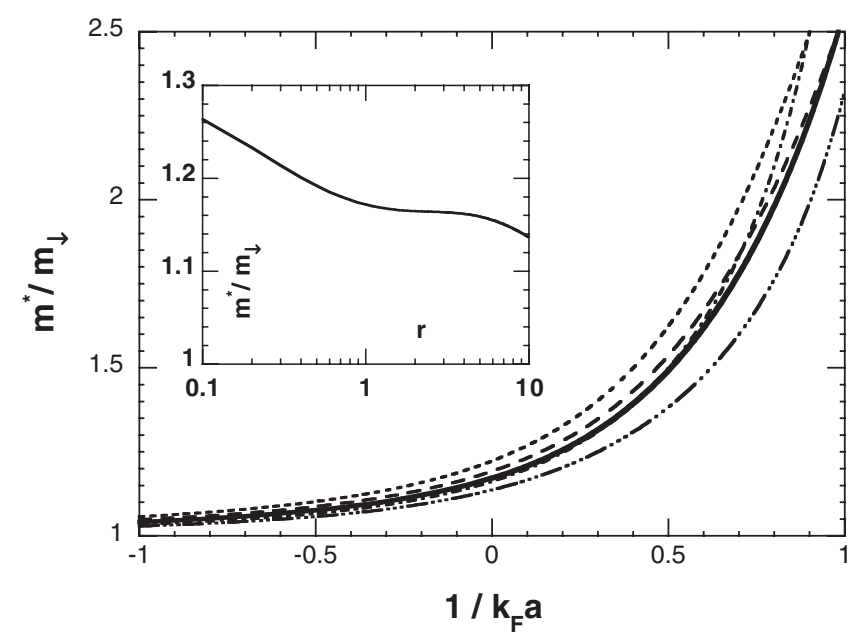

FIG. 2. Relative effective mass $m^{*} / m_{\downarrow}$ as a function of $1 / k_{F} a$ for various mass ratios $r=m_{\downarrow} / m_{\uparrow}$. Same conventions as in Fig. 1 for $r=0.25,0.5$, and 1 . The dashed-dotted line is $r=4$, and the dashed-triple-dotted line is $r=10$. The inset shows $m^{*} / m_{\downarrow}$ as a function of $r$ at unitarity.

$$
\frac{\left|\mu_{\downarrow}\right|}{\mu_{\uparrow}}=\frac{1}{\pi}\left[\left(1+y^{2}\right)\left(\frac{\pi}{2}+\arctan y\right)+y\right],
$$

where $y=1 / k_{F} a$. The result is plotted in Fig. 1 and is seen to be in excellent agreement with the variational and $T$-matrix results. In particular, we find $\rho=0.5$ at unitarity, to be compared to the approximate value 0.465 .

Finally, the relative effective mass $m^{*} / m_{\downarrow}$ is displayed in Fig. 2 for various mass ratios. The first striking feature is that the mass enhancement is quite small around unitarity, whereas we might have expected a much stronger effect around resonance. Naturally, when we go further to the BEC side, $m^{*} / m_{\downarrow}$ increases rapidly. Quantitatively, our result $m^{*} / m_{\downarrow}=1.17$ for equal masses at unitarity is in quite reasonable agreement with the QMC result 1.04(3), taking into account that effective mass is more sensitive to approximations than energy. The other noticeable feature of Fig. 2 is the weak dependence of $m^{*} / m_{\downarrow}$ on the mass ratio $r$, as can be seen in the inset at unitarity. We note that, within our approximation, no bound state appears in the plotted range. We have to keep in mind that, in improved approximations, the location for the appearance of a bound state might be somewhat changed.

To further check the reliability of our approach, we have done the calculations in one dimension. In this case, we can compare our results with the exact solution for equal masses [17]. We find very good agreement along the whole crossover, from BCS-like state to the molecular Tonks state, for the energy, while the mass is more sensitive to our approximations precisely when the two-body bound state plays a major role.

In conclusion, we have found that the physical properties of a single $\downarrow$ atom in the presence of a Fermi sea of $\uparrow$ atoms can be described fairly accurately by the simple inclusion of single particle-hole excitations. Two equiva- lent schemes based, respectively, on a many-body trial wave function and a simple $T$-matrix approach were developed and give very good agreement with known QMC results for $m_{\uparrow}=m_{\downarrow}$ at unitarity.

We are grateful to S. Giorgini, M. Yu. Kagan, $\mathrm{X}$. Leyronas, and S. Stringari for stimulating discussions. The Laboratoire de Physique Statistique and Laboratoire Kastler Brossel are Laboratoires associés au Centre National de la Recherche Scientifique et aux Universités Paris 6 et Paris 7. C. L. and A. R. acknowledge support by the Ministero dell'Istruzione, dell'Università e della Ricerca (MIUR). F. C. acknowledges support from Region Ile de France (IFRAF).

[1] R. Casalbuoni and G. Nardulli, Rev. Mod. Phys. 76, 263 (2004).

[2] M.W. Zwierlein, A. Schirotzek, C.H. Schunck, and W. Ketterle, Science 311, 492 (2006); G. B. Partridge, W. Li, R. I. Kamar, Y. Liao, and R. G. Hulet, Science 311, 503 (2006); Y. Shin, M.W. Zwierlein, C.H. Schunck, A. Schirotzek, and W. Ketterle, Phys. Rev. Lett. 97, 030401 (2006); C.H. Schunk, Y. Shin, A. Schirotzek, M.W. Zwierlein, and W. Ketterle, condmat/0702066.

[3] See, for example, D. E. Sheehy and L. Radzihovsky, condmat/0607803 [Ann. Phys. (N.Y.) (to be published)], and references therein.

[4] A. Bulgac and M. M. Forbes, Phys. Rev. A 75, 031605(R) (2007).

[5] F. Chevy, Phys. Rev. A 74, 063628 (2006).

[6] C. Lobo, A. Recati, S. Giorgini, and S. Stringari, Phys. Rev. Lett. 97, 200403 (2006).

[7] G. D. Mahan, Many-Particle Physics (Kluwer Academic, Dordrecht, 2000).

[8] N. V. Prokof'ev, Phys. Rev. Lett. 74, 2748 (1995), and references therein.

[9] F. Arias de Saavedra, J. Boronat, A. Polls, and A. Fabrocini, Phys. Rev. B 50, 4248 (1994), and references therein.

[10] M. Yu. Kagan, R. Frésard, M. Capezzali, and H. Beck, Phys. Rev. B 57, 5995 (1998), and references therein.

[11] P. Pieri and G. C. Strinati, Phys. Rev. B 61, 15370 (2000), and references therein.

[12] R. Combescot, X. Leyronas, and M. Yu. Kagan, Phys. Rev. A 73, 023618 (2006), and references therein.

[13] S. Pilati and S. Giorgini (unpublished).

[14] For an introduction to many-body techniques, see, for example, A. A. Abrikosov, L. P. Gorkov, and I.E. Dzyaloshinkski, Methods of Quantum Field Theory in Statistical Physics (Dover, New York, 1963).

[15] Reference [13] has calculated $\rho$ on the $a<0$ side for equal masses, and the results are in excellent agreement with ours. The agreement is not as good for the effective mass but still within $10 \%$ in the worst case.

[16] J. Friedel, Philos. Mag. 43, 153 (1952); F. G. Fumi, Philos. Mag. 46, 1007 (1955).

[17] J. B. McGuire, J. Math. Phys. (N.Y.) 7, 123 (1966). 